\title{
On positive solutions of a class of second order nonlinear differential equations on the halfline
}

\author{
by Svatoslav StaněK (Olomouc)
}

\begin{abstract}
The differential equation of the form $\left(q(t) k(u)\left(u^{\prime}\right)^{a}\right)^{\prime}=f(t) h(u) u^{\prime}, a \in$ $(0, \infty)$, is considered and solutions $u$ with $u(0)=0$ and $(u(t))^{2}+\left(u^{\prime}(t)\right)^{2}>0$ on $(0, \infty)$ are studied. Theorems about existence, uniqueness, boundedness and dependence of solutions on a parameter are given.
\end{abstract}

1. Introduction. In [9] the differential equation $\left(q(t) k(u) u^{\prime}\right)^{\prime}=F(t, u) u^{\prime}$ was considered and the author gave sufficient conditions for the existence and uniqueness of solutions $u$ such that $u(0)=0$ and $(u(t))^{2}+\left(u^{\prime}(t)\right)^{2}>0$ for $t \in(0, \infty)$. This problem is connected with the description of the mathematical model of infiltration of water. For more details see e.g. [3], [4] and [6]. Naturally, a question arises of what are the properties of solutions of the differential equation $\left(q(t) k(u)\left(u^{\prime}\right)^{a}\right)^{\prime}=F(t, u) u^{\prime}$, where $a$ is a positive constant. For the sake of simplicity of our assumptions, results and proofs we will consider the differential equations of the type

$$
\left(q(t) k(u)\left(u^{\prime}\right)^{a}\right)^{\prime}=f(t) h(u) u^{\prime}, \quad a \in(0, \infty) .
$$

We also study the qualitative dependence of solutions of (1) on the parameter $a$. As special cases we obtain results of [9] (with $F(t, u)=f(t) h(u)$ and $a=1$ ), of [8] (where $a=1, f \in C^{1}\left(\mathbb{R}_{+}\right), \mathbb{R}_{+}=[0, \infty)$ ) and of [7] (where $a=1, q(t) \equiv 1, h(u) \equiv 1$ ). We observe that special cases of (1) (with $a=1$ ) were also considered in [1], [2], [4] and [6].

2. Notations and lemmas. We consider equation (1) in which the functions $q, k, f$ and $h$ satisfy the following assumptions:

1991 Mathematics Subject Classification: 34A15, 34C11, 34B15, 45G10.

Key words and phrases: nonlinear second order differential equation, nonnegative solution, existence and uniqueness of solutions, bounded solution, dependence of solutions on the parameter, boundary value problem on a noncompact interval, Tikhonov-Schauder fixed point theorem.

Supported by grant no. 201/93/2311 of the Grant Agency of Czech Republic. 
$\left(\mathrm{H}_{1}\right) \quad q \in C^{0}\left(\mathbb{R}_{+}\right), q(t)>0$ for $t>0$ and $\int_{0}(1 / q(s))^{1 / a} d s<\infty$;

$\left(\mathrm{H}_{2}\right) \quad k \in C^{0}\left(\mathbb{R}_{+}\right), k(0)=0, k(u)>0$ for $u>0$ and $\int_{0}(k(s))^{1 / a} d s<\infty$, $\int^{\infty}(k(s))^{1 / a} d s=\infty$;

$\left(\mathrm{H}_{3}\right) \quad f \in C^{0}\left(\mathbb{R}_{+}\right), f(t)>0$ for $t \in \mathbb{R}_{+}$and $f$ is decreasing on $\mathbb{R}_{+}$;

$\left(\mathrm{H}_{4}\right) \quad h \in C^{0}\left(\mathbb{R}_{+}\right), h(u) \geq 0$ for $u \in \mathbb{R}_{+}$and $H(u)=\int_{0}^{u} h(s) d s$ is strictly increasing on $\mathbb{R}_{+}$;

$\left(\mathrm{H}_{5}\right) \quad \int_{0}(k(s) / H(s))^{1 / a} d s<\infty, \int^{\infty}(k(s) / H(s))^{1 / a} d s=\infty$.

We say that $u$ is a solution of (1) if $u \in C^{0}\left(\mathbb{R}_{+}\right) \cap C^{1}((0, \infty)), u(0)=0$, $u(t) \geq 0$ on $\mathbb{R}_{+},(u(t))^{2}+\left(u^{\prime}(t)\right)^{2}>0$ for $t \in(0, \infty), q(t) k(u(t))\left(u^{\prime}(t)\right)^{a}$ is continuously differentiable on $(0, \infty), \lim _{t \rightarrow 0_{+}} q(t) k(u(t))\left(u^{\prime}(t)\right)^{a}=0$ and (1) is satisfied on $(0, \infty)$.

Let $p \in C^{0}(\mathbb{R}), p(0)=0$. We say that $u$ is a solution of the differential equation

$$
\left(q(t) k(u) p\left(u^{\prime}\right)\right)^{\prime}=f(t) h(u) u^{\prime}
$$

if $u \in C^{0}\left(\mathbb{R}_{+}\right) \cap C^{1}((0, \infty)), u(0)=0, u(t) \geq 0$ on $\mathbb{R}_{+},(u(t))^{2}+\left(u^{\prime}(t)\right)^{2}>0$ for $t \in(0, \infty), q(t) k(u(t)) p\left(u^{\prime}(t)\right)$ is continuously differentiable on $(0, \infty)$, $\lim _{t \rightarrow 0_{+}} q(t) k(u(t)) p\left(u^{\prime}(t)\right)=0$ and $(2)$ is satisfied on $(0, \infty)$.

Lemma 1. Let $u(t)$ be a solution of (2). Then $u^{\prime}(t)>0$ for $t \in(0, \infty)$.

Pro of. We see that

$$
q(t) k(u(t)) p\left(u^{\prime}(t)\right)=\int_{0}^{t} f(s) h(u(s)) u^{\prime}(s) d s \quad \text { for } t>0 .
$$

Suppose that there exist $0<t_{1}<t_{2}$ such that $u^{\prime}\left(t_{1}\right)=u^{\prime}\left(t_{2}\right)=0$ and $u^{\prime}(t)>0\left(\right.$ resp. $\left.u^{\prime}(t)<0\right)$ on $\left(t_{1}, t_{2}\right)$. Then $u(t)>0$ for $t \in\left[t_{1}, t_{2}\right]$ and $(3)$ implies

$0=q\left(t_{2}\right) k\left(u\left(t_{2}\right)\right) p\left(u^{\prime}\left(t_{2}\right)\right)-q\left(t_{1}\right) k\left(u\left(t_{1}\right)\right) p\left(u^{\prime}\left(t_{1}\right)\right)=\int_{t_{1}}^{t_{2}} f(s) h(u(s)) u^{\prime}(s) d s$,

which contradicts

$$
\begin{gathered}
\int_{t_{1}}^{t_{2}} f(s) h(u(s)) u^{\prime}(s) d s \geq f\left(t_{2}\right) \int_{u\left(t_{1}\right)}^{u\left(t_{2}\right)} h(s) d s>0 \\
\text { (resp. } \left.\int_{t_{1}}^{t_{2}} f(s) h(u(s)) u^{\prime}(s) d s \leq f\left(t_{2}\right) \int_{u\left(t_{1}\right)}^{u\left(t_{2}\right)} h(s) d s<0\right) .
\end{gathered}
$$

Assume $u^{\prime}(\tau)=0$ for a $\tau \in(0, \infty)$ and $u^{\prime}(t) \neq 0$ on $(0, \tau)$. Then necessarily 
$u^{\prime}(t)>0$ on $(0, \tau)$ since $u(t) \geq 0$ for $t \in \mathbb{R}_{+}$, and (cf. (3))

$$
0=q(\tau) k(u(\tau)) p\left(u^{\prime}(\tau)\right)=\int_{0}^{\tau} f(s) h(u(s)) u^{\prime}(s) d s,
$$

which contradicts

$$
\int_{0}^{\tau} f(s) h(u(s)) u^{\prime}(s) d s \geq f(\tau) \int_{0}^{u(\tau)} h(s) d s>0 .
$$

Therefore by virtue of $(u(t))^{2}+\left(u^{\prime}(t)\right)^{2}>0$ on $(0, \infty)$ we conclude $u^{\prime}(t)>0$ for $t \in(0, \infty)$.

Corollary 1. Let $u(t)$ be a solution of (1). Then $u^{\prime}(t)>0$ for $t \in$ $(0, \infty)$.

Proof. If $a=m / n$, where $m, n \in \mathbb{N}$ and $n$ is odd, then the function $v^{a}$ is defined for all $v \in \mathbb{R}$ and Corollary 1 follows from Lemma 1. Assume $a=m / n$, where $m, n \in \mathbb{N}$ and $n$ is even or $a$ is an irrational number. Then the function $v^{a}$ is defined for all $v \in \mathbb{R}_{+}$, and for every $p_{1} \in C^{0}((-\infty, 0])$ with $p_{1}(0)=0$, the function $p: \mathbb{R} \rightarrow \mathbb{R}$ defined by $p(v)=v^{a}$ for $v \in \mathbb{R}_{+}$and $p(v)=p_{1}(v)$ for $v \in(-\infty, 0)$ is continuous on $\mathbb{R}, p(0)=0$ and, moreover, $u(t)$ is a solution of $(2)$. Hence $u^{\prime}(t)>0$ on $(0, \infty)$ by Lemma 1 .

Remark 1. It follows from Corollary 1 that $u \in \mathcal{A}$ for any solution $u$ of (1), where

$$
\mathcal{A}=\left\{u \in C^{0}\left(\mathbb{R}_{+}\right): u(0)=0, u \text { is strictly increasing on } \mathbb{R}_{+}\right\} .
$$

Set

$$
k_{1}(u)=(k(u))^{1 / a}, \quad K_{1}(u)=\int_{0}^{u} k_{1}(s) d s, \quad P(u)=\int_{0}^{u}\left(\frac{k(s)}{H(s)}\right)^{1 / a} d s
$$

for $u \in \mathbb{R}_{+}$. Obviously, $k_{1} \in C^{0}\left(\mathbb{R}_{+}\right), K_{1} \in C^{1}\left(\mathbb{R}_{+}\right), P \in C^{0}\left(\mathbb{R}_{+}\right) \cap$ $C^{1}((0, \infty)), K_{1}$ and $P$ are strictly increasing on $\mathbb{R}_{+}, \lim _{u \rightarrow \infty} K_{1}(u)=\infty$ by $\left(\mathrm{H}_{2}\right)$ and $\lim _{u \rightarrow \infty} P(u)=\infty$ by $\left(\mathrm{H}_{5}\right)$.

LEMma 2. If $u(t)$ is a solution of (1), then

$$
u(t)=K_{1}^{-1}\left(\int_{0}^{t}\left(\frac{1}{q(s)} \int_{0}^{u(s)} f\left(u^{-1}(\tau)\right) h(\tau) d \tau\right)^{1 / a} d s\right), \quad t \in \mathbb{R}_{+},
$$

where $K_{1}^{-1}$ and $u^{-1}$ denote the inverse functions to $K_{1}$ and $u$, respectively. Conversely, if $u \in \mathcal{A}$ is a solution of (4), then $u(t)$ is a solution of (1).

Pro of. Let $u$ be a solution of (1). Then $u \in \mathcal{A}$ (cf. Remark 1) and

$$
\left(k_{1}(u(t)) u^{\prime}(t)\right)^{a}=\frac{1}{q(t)} \int_{0}^{t} f(s) h(u(s)) u^{\prime}(s) d s, \quad t>0 .
$$


Hence

$$
\left(K_{1}(u(t))\right)^{\prime}=\left(\frac{1}{q(t)} \int_{0}^{u(t)} f\left(u^{-1}(s)\right) h(s) d s\right)^{1 / a}, \quad t>0,
$$

and integrating (5) from 0 to $t$, we obtain

$$
K_{1}(u(t))=\int_{0}^{t}\left(\frac{1}{q(s)} \int_{0}^{u(s)} f\left(u^{-1}(\tau)\right) h(\tau) d \tau\right)^{1 / a} d s, \quad t \in \mathbb{R}_{+},
$$

and consequently, equality (4) is satisfied.

Conversely, let $u \in \mathcal{A}$ be a solution of (4). Then $u \in C^{1}((0, \infty))$,

$$
\lim _{t \rightarrow 0_{+}} q(t) k(u(t))\left(u^{\prime}(t)\right)^{a}=\lim _{t \rightarrow 0_{+}} \int_{0}^{u(t)} f\left(u^{-1}(s)\right) h(s) d s=0
$$

and $\left(q(t) k(u(t))\left(u^{\prime}(t)\right)^{a}\right)^{\prime}=f(t) h(u(t)) u^{\prime}(t)$ for $t \in(0, \infty)$. Hence $u$ is a solution of $(1)$.

Define $\underline{\varphi}, \bar{\varphi}: \mathbb{R}_{+} \rightarrow \mathbb{R}_{+}$by

$$
\underline{\varphi}(t)=P^{-1}\left(\int_{0}^{t}\left(\frac{f(s)}{q(s)}\right)^{1 / a} d s\right), \quad \bar{\varphi}(t)=P^{-1}\left(\int_{0}^{t}\left(\frac{f(0)}{q(s)}\right)^{1 / a} d s\right),
$$

where $P^{-1}: \mathbb{R}_{+} \rightarrow \mathbb{R}_{+}$denotes the inverse function to $P$. Obviously, $\underline{\varphi}(t) \leq$ $\bar{\varphi}(t)$ on $\mathbb{R}_{+}$by $\left(\mathrm{H}_{3}\right)$.

LEMma 3. Let $u(t)$ be a solution of (1). Then

(6)

$$
\underline{\varphi}(t) \leq u(t) \leq \bar{\varphi}(t) \quad \text { for } t \in \mathbb{R}_{+} .
$$

Proof. Since

$$
\begin{aligned}
f(t) H(u(t)) & =f(t) \int_{0}^{u(t)} h(s) d s \leq \int_{0}^{t} f(s) h(u(s)) u^{\prime}(s) d s \\
& \leq f(0) \int_{0}^{u(t)} h(s) d s=f(0) H(u(t)),
\end{aligned}
$$

we have

$$
f(t) H(u(t)) \leq q(t)\left(k_{1}(u(t)) u^{\prime}(t)\right)^{a} \leq f(0) H(u(t)), \quad t \in(0, \infty) .
$$

Thus

and

$$
\left(\frac{f(t)}{q(t)} H(u(t))\right)^{1 / a} \leq k_{1}(u(t)) u^{\prime}(t) \leq\left(\frac{f(0)}{q(t)} H(u(t))\right)^{1 / a}
$$


(7) $\left(\frac{f(t)}{q(t)}\right)^{1 / a} \leq\left(\frac{k(u(t))}{H(u(t))}\right)^{1 / a} u^{\prime}(t)\left(=(P(u(t)))^{\prime}\right) \leq\left(\frac{f(0)}{q(t)}\right)^{1 / a}$,

$t \in(0, \infty)$.

Integrating (7) from 0 to $t$ we obtain

$$
\int_{0}^{t}\left(\frac{f(s)}{q(s)}\right)^{1 / a} d s \leq P(u(t)) \leq \int_{0}^{t}\left(\frac{f(0)}{q(s)}\right)^{1 / a} d s, \quad t \in \mathbb{R}_{+},
$$

and (6) holds.

Set

$$
\begin{aligned}
& \mathcal{K}=\left\{u \in \mathcal{A}: \underline{\varphi}(t) \leq u(t) \leq \bar{\varphi}(t) \text { for } t \in \mathbb{R}_{+}\right. \text {and } \\
& u\left(t_{2}\right)-u\left(t_{1}\right) \geq\left(f\left(t_{2}\right) H\left(\underline{\varphi}\left(t_{1}\right)\right)\right)^{1 / a} \int_{t_{1}}^{t_{2}}(1 / q(s))^{1 / a} d s \\
&\left.\times\left[\max \left\{k_{1}(u): \underline{\varphi}\left(t_{1}\right) \leq u \leq \bar{\varphi}\left(t_{2}\right)\right\}\right]^{-1} \text { for } 0<t_{1}<t_{2}\right\} .
\end{aligned}
$$

Remark 2. We now verify that $\underline{\varphi} \in \mathcal{K}$ and thus $\mathcal{K}$ is a nonempty subset of $\mathcal{A}$. Fix $0<t_{1}<t_{2}$. Then

$$
P\left(\underline{\varphi}\left(t_{2}\right)\right)-P\left(\underline{\varphi}\left(t_{1}\right)\right)=\int_{t_{1}}^{t_{2}}\left(\frac{f(s)}{q(s)}\right)^{1 / a} d s
$$

and, by the Taylor formula, there exists $\xi \in\left(\underline{\varphi}\left(t_{1}\right), \underline{\varphi}\left(t_{2}\right)\right) \subset\left(\underline{\varphi}\left(t_{1}\right), \bar{\varphi}\left(t_{2}\right)\right)$ such that

$$
P^{\prime}(\xi)\left(\underline{\varphi}\left(t_{2}\right)-\underline{\varphi}\left(t_{1}\right)\right) \geq\left(f\left(t_{2}\right)\right)^{1 / a} \int_{t_{1}}^{t_{2}}\left(\frac{1}{q(s)}\right)^{1 / a} d s .
$$

Since

$$
P^{\prime}(\xi)=\frac{k_{1}(\xi)}{(H(\xi))^{1 / a}} \leq \max \left\{k_{1}(u): \underline{\varphi}\left(t_{1}\right) \leq u \leq \bar{\varphi}\left(t_{2}\right)\right\}\left(\frac{1}{H\left(\underline{\varphi}\left(t_{1}\right)\right)}\right)^{1 / a},
$$

we get

$$
\begin{aligned}
\underline{\varphi}\left(t_{2}\right)-\underline{\varphi}\left(t_{1}\right) \geq & \frac{1}{P^{\prime}(\xi)}\left(f\left(t_{2}\right)\right)^{1 / a} \int_{t_{1}}^{t_{2}}\left(\frac{1}{q(s)}\right)^{1 / a} d s \\
\geq & \left(f\left(t_{2}\right) H\left(\underline{\varphi}\left(t_{1}\right)\right)\right)^{1 / a} \int_{t_{1}}^{t_{2}}\left(\frac{1}{q(s)}\right)^{1 / a} d s \\
& \times\left[\max \left\{k_{1}(u): \underline{\varphi}\left(t_{1}\right) \leq u \leq \bar{\varphi}\left(t_{2}\right)\right\}\right]^{-1}
\end{aligned}
$$

and therefore $\underline{\varphi} \in \mathcal{K}$. Analogously we can show that $\bar{\varphi} \in \mathcal{K}$ as well. 
Define the operator $\mathrm{T}: \mathcal{K} \rightarrow C^{0}\left(\mathbb{R}_{+}\right)$by

$$
(\mathrm{T} u)(t)=K_{1}^{-1}\left(\int_{0}^{t}\left(\frac{1}{q(s)} \int_{0}^{u(s)} f\left(u^{-1}(\tau)\right) h(\tau) d \tau\right)^{1 / a} d s\right), \quad t \in \mathbb{R}_{+} .
$$

LEMMA 4 . T $: \mathcal{K} \rightarrow \mathcal{K}$.

Proof. Let $u \in \mathcal{K}$. Set

$$
\begin{aligned}
\gamma(t) & =\int_{0}^{t}\left(\frac{1}{q(s)} \int_{0}^{u(s)} f\left(u^{-1}(\tau)\right) h(\tau) d \tau\right)^{1 / a} d s, \\
\alpha(t) & =\gamma(t)-K_{1}(\underline{\varphi}(t)), \quad \beta(t)=\gamma(t)-K_{1}(\bar{\varphi}(t))
\end{aligned}
$$

for $t \in \mathbb{R}_{+}$. Then

$$
\begin{aligned}
\alpha^{\prime}(t) & =\left(\frac{1}{q(t)} \int_{0}^{u(t)} f\left(u^{-1}(s)\right) h(s) d s\right)^{1 / a}-\frac{k_{1}(\underline{\varphi}(t))}{P^{\prime}(\underline{\varphi}(t))}\left(\frac{f(t)}{q(t)}\right)^{1 / a} \\
& \geq\left(\frac{f(t)}{q(t)} H(u(t))\right)^{1 / a}-\frac{k_{1}(\underline{\varphi}(t))}{k_{1}(\underline{\varphi}(t))}\left(\frac{f(t)}{q(t)} H(\underline{\varphi}(t))\right)^{1 / a} \geq 0, \\
\beta^{\prime}(t) & =\left(\frac{1}{q(t)} \int_{0}^{u(t)} f\left(u^{-1}(s)\right) h(s) d s\right)^{1 / a}-\frac{k_{1}(\bar{\varphi}(t))}{P^{\prime}(\bar{\varphi}(t))}\left(\frac{f(0)}{q(t)}\right)^{1 / a} \\
& \leq\left(\frac{f(0)}{q(t)} H(u(t))\right)^{1 / a}-\frac{k_{1}(\bar{\varphi}(t))}{k_{1}(\bar{\varphi}(t))}\left(\frac{f(0)}{q(t)} H(\bar{\varphi}(t))\right)^{1 / a} \leq 0
\end{aligned}
$$

for $t \in(0, \infty)$. Since $\alpha(0)=\beta(0)=0$ and $\alpha^{\prime}(t) \geq 0, \beta^{\prime}(t) \leq 0$ on $(0, \infty)$, we see that $\alpha(t) \geq 0, \beta(t) \leq 0$ for $t \in \mathbb{R}_{+}$, and consequently,

$$
\underline{\varphi}(t) \leq K_{1}^{-1}(\gamma(t))=(\mathrm{T} u)(t) \leq \bar{\varphi}(t) \quad \text { for } t \in \mathbb{R}_{+} .
$$

Let $0<t_{1}<t_{2}$. Then

$$
\begin{aligned}
K_{1}\left((\mathrm{~T} u)\left(t_{2}\right)\right)-K_{1}\left((\mathrm{~T} u)\left(t_{1}\right)\right) & =\int_{t_{1}}^{t_{2}}\left(\frac{1}{q(s)} \int_{0}^{u(s)} f\left(u^{-1}(\tau)\right) h(\tau) d \tau\right)^{1 / a} d s \\
& \geq \int_{t_{1}}^{t_{2}}\left(\frac{f(s)}{q(s)} H(u(s))\right)^{1 / a} d s \\
& \geq\left(H\left(\underline{\varphi}\left(t_{1}\right)\right) f\left(t_{2}\right)\right)^{1 / a} \int_{t_{1}}^{t_{2}}\left(\frac{1}{q(s)}\right)^{1 / a} d s
\end{aligned}
$$

and 


$$
\begin{aligned}
K_{1}\left((\mathrm{~T} u)\left(t_{2}\right)\right) & -K_{1}\left((\mathrm{~T} u)\left(t_{1}\right)\right) \\
& =k_{1}(\xi)\left[(\mathrm{T} u)\left(t_{2}\right)-(\mathrm{T} u)\left(t_{1}\right)\right] \\
& \leq \max \left\{k_{1}(u): \underline{\varphi}\left(t_{1}\right) \leq u \leq \bar{\varphi}\left(t_{2}\right)\right\}\left[(\mathrm{T} u)\left(t_{2}\right)-(\mathrm{T} u)\left(t_{1}\right)\right]
\end{aligned}
$$

by the Taylor formula (here $\left.\xi \in\left((\mathrm{T} u)\left(t_{1}\right),(\mathrm{T} u)\left(t_{2}\right)\right) \subset\left(\varphi\left(t_{1}\right), \bar{\varphi}\left(t_{2}\right)\right)\right)$. Hence (with $A=\left[\max \left\{k_{1}(u): \underline{\varphi}\left(t_{1}\right) \leq u \leq \bar{\varphi}\left(t_{2}\right)\right\}\right]^{-1}$ )

$$
\begin{aligned}
(\mathrm{T} u)\left(t_{2}\right)-(\mathrm{T} u)\left(t_{1}\right) & \geq A\left[K_{1}\left((\mathrm{~T} u)\left(t_{2}\right)\right)-K_{1}\left((\mathrm{~T} u)\left(t_{1}\right)\right)\right] \\
& \geq A\left(H\left(\underline{\varphi}\left(t_{1}\right)\right) f\left(t_{2}\right)\right)^{1 / a} \int_{t_{1}}^{t_{2}}\left(\frac{1}{q(s)}\right)^{1 / a} d s .
\end{aligned}
$$

From (8) and (9) it follows that $\mathrm{T} u \in \mathcal{K}$ for each $u \in \mathcal{K}$, and consequently, $\mathrm{T}: \mathcal{K} \rightarrow \mathcal{K}$.

\section{Existence theorem}

Theorem 1. Let assumptions $\left(\mathrm{H}_{1}\right)-\left(\mathrm{H}_{5}\right)$ be satisfied. Then there exists a solution of (1).

Proof. By Lemma 2 and Corollary $1, u \in \mathcal{A}$ is a solution of (1) if and only if $u$ is a solution of (4). Therefore in order to prove Theorem 1 it is enough to show that the operator $\mathrm{T}$ has a fixed point.

Let $\mathbf{X}$ be the Fréchet space of $C^{0}$-functions on $\mathbb{R}_{+}$with the topology of uniform convergence on compact subintervals of $\mathbb{R}_{+}$. Then $\mathcal{K}$ is a bounded closed convex subset of $\mathbf{X}$ and $\mathrm{T}: \mathcal{K} \rightarrow \mathcal{K}$ (by Lemma 4). Let $\left\{u_{n}\right\} \subset \mathcal{K}$ be a convergent sequence, $\lim _{n \rightarrow \infty} u_{n}=u(\in \mathcal{K})$. Then $\lim _{n \rightarrow \infty} u_{n}^{-1}=$ $u^{-1}\left(u_{n}^{-1}\right.$ and $u^{-1}$ denote the inverse functions to $u_{n}$ and $u$, respectively) and consequently, $\lim _{n \rightarrow \infty} \mathrm{T} u_{n}=\mathrm{T} u$. This proves that $\mathrm{T}$ is a continuous operator.

It follows from the inequalities $\left(0 \leq t_{1}<t_{2} \leq t_{3}, u \in \mathcal{K}\right)$

$$
\begin{aligned}
(0 \leq) K_{1}\left((\mathrm{~T} u)\left(t_{2}\right)\right)-K_{1}\left((\mathrm{~T} u)\left(t_{1}\right)\right) & \\
& =\int_{t_{1}}^{t_{2}}\left(\frac{1}{q(s)} \int_{0}^{u(s)} f\left(u^{-1}(\tau)\right) h(\tau) d \tau\right)^{1 / a} d s \\
& \leq \int_{t_{1}}^{t_{2}}\left(\frac{f(0)}{q(s)} H(u(s))\right)^{1 / a} d s \\
& \leq\left(f(0) H\left(\bar{\varphi}\left(t_{3}\right)\right)\right)^{1 / a} \int_{t_{1}}^{t_{2}}\left(\frac{1}{q(s)}\right)^{1 / a} d s
\end{aligned}
$$

and from the Arzelà-Ascoli theorem that $\mathrm{T}(\mathcal{K})$ is a relatively compact subset of $\mathcal{K}$. By the Tikhonov-Schauder fixed point theorem, there exists a fixed point of T. Hence Theorem 1 is proved. 
THEOREM 2. Let assumptions $\left(\mathrm{H}_{1}\right)-\left(\mathrm{H}_{5}\right)$ be satisfied. If there exist two different solutions $u(t)$ and $v(t)$ of $(1)$ then

$$
u(t) \neq v(t) \quad \text { for } t \in(0, \infty) .
$$

P r o of. Assume $u, v$ are different solutions of (1). Assume there exists a $t_{1}>0$ such that $u\left(t_{1}\right)=v\left(t_{1}\right)$ and $u(t) \neq v(t)$ on $\left(0, t_{1}\right)$, say $u(t)<v(t)$ for $t \in\left(0, t_{1}\right)$. Then

$$
\begin{aligned}
0= & v\left(t_{1}\right)-u\left(t_{1}\right)=K_{1}\left((\mathrm{~T} v)\left(t_{1}\right)\right)-K_{1}\left((\mathrm{~T} u)\left(t_{1}\right)\right) \\
= & \int_{0}^{t_{1}}\left(\frac{1}{q(s)} \int_{0}^{v(s)} f\left(v^{-1}(\tau)\right) h(\tau) d \tau\right)^{1 / a} d s \\
& -\int_{0}^{t_{1}}\left(\frac{1}{q(s)} \int_{0}^{u(s)} f\left(u^{-1}(\tau)\right) h(\tau) d \tau\right)^{1 / a} d s,
\end{aligned}
$$

which contradicts

$$
\begin{aligned}
\int_{0}^{t_{1}}\left(\frac{1}{q(s)} \int_{0}^{v(s)} f\left(v^{-1}(\tau)\right) h(\tau) d \tau\right)^{1 / a} d s & \\
& >\int_{0}^{t_{1}}\left(\frac{1}{q(s)} \int_{0}^{u(s)} f\left(u^{-1}(\tau)\right) h(\tau) d \tau\right)^{1 / a} d s
\end{aligned}
$$

Let $0<t_{1}<t_{2}$ be such that $u\left(t_{1}\right)=v\left(t_{1}\right), u\left(t_{2}\right)=v\left(t_{2}\right), u(t) \neq v(t)$ on $\left(t_{1}, t_{2}\right)$, say $u(t)>v(t)$ for $t \in\left(t_{1}, t_{2}\right)$. Then $u^{\prime}\left(t_{1}\right) \geq v^{\prime}\left(t_{1}\right), u^{\prime}\left(t_{2}\right) \leq v^{\prime}\left(t_{2}\right)$ and

$$
\begin{aligned}
0 \leq & q\left(t_{1}\right) k\left(u\left(t_{1}\right)\right)\left(\left(u^{\prime}\left(t_{1}\right)\right)^{a}-\left(v^{\prime}\left(t_{1}\right)\right)^{a}\right) \\
& -q\left(t_{2}\right) k\left(u\left(t_{2}\right)\right)\left(\left(u^{\prime}\left(t_{2}\right)\right)^{a}-\left(v^{\prime}\left(t_{2}\right)\right)^{a}\right) \\
= & \int_{t_{2}}^{t_{1}} f(s) h(u(s)) u^{\prime}(s) d s-\int_{t_{2}}^{t_{1}} f(s) h(v(s)) v^{\prime}(s) d s \\
= & \int_{u\left(t_{2}\right)}^{u\left(t_{1}\right)}\left[f\left(u^{-1}(s)\right)-f\left(v^{-1}(s)\right)\right] h(s) d s .
\end{aligned}
$$

On the other hand, since $u\left(t_{2}\right)>u\left(t_{1}\right)$ and $f\left(u^{-1}(t)\right)-f\left(v^{-1}(t)\right) \geq 0$ on $\left[u\left(t_{1}\right), u\left(t_{2}\right)\right]$,

$$
\int_{u\left(t_{2}\right)}^{u\left(t_{1}\right)}\left[f\left(u^{-1}(s)\right)-f\left(v^{-1}(s)\right)\right] h(s) d s \leq 0 .
$$

Thus by (10), $u^{\prime}\left(t_{1}\right)=v^{\prime}\left(t_{1}\right), u^{\prime}\left(t_{2}\right)=v^{\prime}\left(t_{2}\right)$ and $f\left(u^{-1}(t)\right)=f\left(v^{-1}(t)\right)$ for $t \in\left[u\left(t_{1}\right), u\left(t_{2}\right)\right]$. Since 


$$
\begin{aligned}
& q(t)\left(\left(K_{1}(u(t))\right)^{\prime}\right)^{a}-q\left(t_{1}\right) k\left(u\left(t_{1}\right)\right)\left(u^{\prime}\left(t_{1}\right)\right)^{a}=\int_{u\left(t_{1}\right)}^{u(t)} f\left(u^{-1}(s)\right) h(s) d s, \\
& q(t)\left(\left(K_{1}(v(t))\right)^{\prime}\right)^{a}-q\left(t_{1}\right) k\left(v\left(t_{1}\right)\right)\left(v^{\prime}\left(t_{1}\right)\right)^{a}=\int_{u\left(t_{1}\right)}^{v(t)} f\left(v^{-1}(s)\right) h(s) d s
\end{aligned}
$$

on $(0, \infty), q\left(t_{1}\right) k\left(u\left(t_{1}\right)\right)\left(u^{\prime}\left(t_{1}\right)\right)^{a}=q\left(t_{1}\right) k\left(v\left(t_{1}\right)\right)\left(v^{\prime}\left(t_{1}\right)\right)^{a}, 0<f\left(u^{-1}(s)\right)=$ $f\left(v^{-1}(s)\right)$ for $s \in\left[u\left(t_{1}\right), u\left(t_{2}\right)\right]$ and $u(t)>v(t)$ on $\left(t_{1}, t_{2}\right)$, we obtain

$$
\begin{aligned}
&\left(\left(K_{1}(u(t))\right)^{\prime}\right)^{a}-\left(\left(K_{1}(v(t))\right)^{\prime}\right)^{a} \\
&=\frac{1}{q(t)} \int_{v(t)}^{u(t)} f\left(u^{-1}(s)\right) h(s) d s>0, \quad t \in\left(t_{1}, t_{2}\right) .
\end{aligned}
$$

Thus

$$
\left(K_{1}(u(t))\right)^{\prime}>\left(K_{1}(v(t))\right)^{\prime} \quad \text { for } t \in\left(t_{1}, t_{2}\right),
$$

and consequently, $K_{1}\left(u\left(t_{2}\right)\right)-K_{1}\left(u\left(t_{1}\right)\right)>K_{1}\left(v\left(t_{2}\right)\right)-K_{1}\left(v\left(t_{1}\right)\right)$, which contradicts $u\left(t_{1}\right)=v\left(t_{1}\right), u\left(t_{2}\right)=v\left(t_{2}\right)$. So either $u(t) \neq v(t)$ on $(0, \infty)$ or there exists a $t_{0} \in(0, \infty)$ such that $u(t)=v(t)$ for $t \in\left[0, t_{0}\right]$ and $u(t) \neq v(t)$ on $\left(t_{0}, \infty\right)$, say for example $u(t)>v(t)$ for $t \in\left(t_{0}, \infty\right)$. Assume that the second case occurs. Then, by the Bonnet mean value theorem, there exists a $\xi \in\left[t_{0}, t\right]$ such that

$$
\begin{aligned}
\left(\left(K_{1}(u(t))\right)^{\prime}\right)^{a}- & \left(\left(K_{1}(v(t))\right)^{\prime}\right)^{a} \\
= & \frac{1}{q(t)} \int_{t_{0}}^{t} f(s)\left[h(u(s)) u^{\prime}(s)-h(v(s)) v^{\prime}(s)\right] d s \\
= & \frac{1}{q(t)}\left[f\left(t_{0}\right) \int_{t_{0}}^{\xi}\left(h(u(s)) u^{\prime}(s)-h(v(s)) v^{\prime}(s)\right) d s\right. \\
& \left.+f(t) \int_{\xi}^{t}\left(h(u(s)) u^{\prime}(s)-h(v(s)) v^{\prime}(s)\right) d s\right] \\
= & \frac{1}{q(t)}\left[\left(f\left(t_{0}\right)-f(t)\right)(H(u(\xi))-H(v(\xi)))\right. \\
& +f(t)(H(u(t))-H(v(t)))], \quad t \geq t_{0} .
\end{aligned}
$$

Set

$$
\begin{aligned}
M= & a \min \left\{q(t): t_{0} \leq t \leq t_{0}+1\right\} \cdot \min \left\{\left(k_{1}(z)\right)^{a-1}: u\left(t_{0}\right) \leq z \leq u\left(t_{0}+1\right)\right\} \\
& \times \min \left\{\min \left\{\left(u^{\prime}(t)\right)^{a-1},\left(v^{\prime}(t)\right)^{a-1}\right\}: t_{0} \leq t \leq t_{0}+1\right\}(>0), \\
M_{1}= & \min \left\{k_{1}(z): u\left(t_{0}\right) \leq z \leq u\left(t_{0}+1\right)\right\}(>0)
\end{aligned}
$$




$$
\begin{aligned}
L & =\max \left\{h(z): u\left(t_{0}\right) \leq z \leq u\left(t_{0}+1\right)\right\}(>0), \\
V(t) & =\max \left\{u(s)-v(s): t_{0} \leq s \leq t\right\} \quad \text { for } t \in\left[t_{0}, t_{0}+1\right] .
\end{aligned}
$$

Obviously, $V\left(t_{0}\right)=0$ and $V$ is continuous nondecreasing on $\left[t_{0}, t_{0}+1\right]$.

By the Taylor formula, there exists a $B(=B(t))$ in the interval with end points $\left(K_{1}(u(t))\right)^{\prime}$ and $\left(K_{1}(v(t))\right)^{\prime}$ such that

$$
\begin{aligned}
\left(\left(K_{1}(u(t))\right)^{\prime}\right)^{a}-\left(\left(K_{1}(v(t))\right)^{\prime}\right)^{a}=a B^{a-1}\left(K_{1}(u(t))-K_{1}(v(t))\right)^{\prime}, & \\
t & \in\left[t_{0}, t_{0}+1\right],
\end{aligned}
$$

and therefore (cf. (12))

$$
\begin{aligned}
&\left(K_{1}(u(t))-K_{1}(v(t))\right)^{\prime} \\
& \leq \frac{1}{M}\left[\left(f\left(t_{0}\right)-f(t)\right)(H(u(\xi))-H(v(\xi)))\right. \\
&\quad+f(t)(H(u(t))-H(v(t)))] \\
& \leq \frac{f\left(t_{0}\right)}{M}[(H(u(\xi))-H(v(\xi)))+(H(u(t))-H(v(t)))] \\
& \leq \frac{2}{M} L f\left(t_{0}\right) V(t), \quad t \in\left[t_{0}, t_{0}+1\right] .
\end{aligned}
$$

Then

$$
K_{1}(u(t))-K_{1}(v(t)) \leq \frac{2}{M} L f\left(t_{0}\right) \int_{t_{0}}^{t} V(s) d s,
$$

and consequently,

$$
u(t)-v(t) \leq \frac{2 L f\left(t_{0}\right)}{M k_{1}(\varepsilon)} \int_{t_{0}}^{t} V(s) d s \leq \frac{2 L f\left(t_{0}\right)}{M M_{1}} \int_{t_{0}}^{t} V(s) d s, \quad t \in\left[t_{0}, t_{0}+1\right],
$$

where $\varepsilon \in[v(t), u(t)]$ by the Taylor formula. Hence

$$
\begin{aligned}
V(t) & \leq \frac{2 L f\left(t_{0}\right)}{M M_{1}} \int_{t_{0}}^{t} V(s) d s \leq \frac{2 L f\left(t_{0}\right)}{M M_{1}} V(t) \int_{t_{0}}^{t} d s \\
& =\frac{2 L f\left(t_{0}\right)}{M M_{1}} V(t)\left(t-t_{0}\right), \quad t \in\left[t_{0}, t_{0}+1\right] .
\end{aligned}
$$

Since $V(t)>0$ for $t \in\left(t_{0}, t_{0}+1\right.$ ], we obtain (cf. (13))

$$
1 \leq \frac{2 L f\left(t_{0}\right)}{M M_{1}}\left(t-t_{0}\right) \quad \text { for } t \in\left(t_{0}, t_{0}+1\right],
$$

a contradiction. 
Theorem 3. Let assumptions $\left(\mathrm{H}_{1}\right)-\left(\mathrm{H}_{5}\right)$ be satisfied. Then there exist solutions $\underline{u}(t)$ and $\bar{u}(t)$ of (1) such that

$$
\underline{\varphi}(t) \leq \underline{u}(t) \leq u(t) \leq \bar{u}(t) \leq \bar{\varphi}(t), \quad t \in \mathbb{R}_{+},
$$

for any solution $u(t)$ of (1).

Pr o of. Denote by $\mathcal{B}$ the set of all solutions of (1). By Theorem $1, \mathcal{B}$ is a nonempty set. If $\mathcal{B}$ is a finite set, then Theorem 3 follows from Theorem 2. Assume $\mathcal{B}$ is an infinite set. Set

$$
\underline{u}(t)=\inf \{u(t): u \in \mathcal{B}\}, \quad \bar{u}(t)=\sup \{u(t): u \in \mathcal{B}\} \quad \text { for } t \in \mathbb{R}_{+} .
$$

Then $\underline{\varphi}(t) \leq \underline{u}(t) \leq \bar{u}(t) \leq \bar{\varphi}(t)$ on $\mathbb{R}_{+}$and to prove Theorem 3 it is enough to show that $\underline{u}$ and $\bar{u}$ are solutions of (1). By Theorem 2, there exists a sequence $\left\{u_{n}\right\} \subset \mathcal{B}, u_{1}(t)<\ldots<u_{n}(t)<\ldots<\bar{u}(t), t \in(0, \infty)$, such that $\bar{u}(t)=\lim _{n \rightarrow \infty} u_{n}(t)$ for $t \in \mathbb{R}_{+}$. Now we prove that $\lim _{n \rightarrow \infty} u_{n}^{\prime}(t)=: b(t)$ exists for all $t \in(0, \infty)$ and $b=\bar{u}^{\prime}$. Evidently,

$$
\begin{aligned}
& \left(K_{1}\left(u_{n+1}(t)\right)\right)^{\prime}-\left(K_{1}\left(u_{n}(t)\right)\right)^{\prime} \\
& =\left(\frac{1}{q(t)} \int_{0}^{u_{n+1}(t)} f\left(u_{n+1}^{-1}(s)\right) h(s) d s\right)^{1 / a}-\left(\frac{1}{q(t)} \int_{0}^{u_{n}(t)} f\left(u_{n}^{-1}(s)\right) h(s) d s\right)^{1 / a} \\
& >\left(\frac{1}{q(t)} \int_{0}^{u_{n}(t)} f\left(u_{n}^{-1}(s)\right) h(s) d s\right)^{1 / a}-\left(\frac{1}{q(t)} \int_{0}^{u_{n}(t)} f\left(u_{n}^{-1}(s)\right) h(s) d s\right)^{1 / a}=0
\end{aligned}
$$

for $t \in(0, \infty)$ and $n \in \mathbb{N}$. Therefore the sequence $\left\{k_{1}\left(u_{n}(t)\right) u_{n}^{\prime}(t)\right\}$ is strictly increasing for each $t \in(0, \infty)$. Setting $\alpha(t)=\lim _{n \rightarrow \infty} k_{1}\left(u_{n}(t)\right) u_{n}^{\prime}(t), t \in$ $(0, \infty)$, we see that

$$
\lim _{n \rightarrow \infty} u_{n}^{\prime}(t)=\lim _{n \rightarrow \infty} \frac{k_{1}\left(u_{n}(t)\right) u_{n}^{\prime}(t)}{k_{1}\left(u_{n}(t)\right)}=\frac{\alpha(t)}{k_{1}(\bar{u}(t))}=: \beta(t), \quad t \in(0, \infty),
$$

and using the Lebesgue dominated convergence theorem in the equalities

$$
u_{n}(t)=\int_{0}^{t} u_{n}^{\prime}(s) d s, \quad t \in \mathbb{R}_{+}, n \in \mathbb{N},
$$

we get $\bar{u}(t)=\int_{0}^{t} \beta(s) d s$ on $\mathbb{R}_{+}$; hence $\beta(t)=\bar{u}^{\prime}(t)$ for $t \in(0, \infty)$. Applying again the Lebesgue theorem to the equalities

$$
k_{1}\left(u_{n}(t)\right) u_{n}^{\prime}(t)=\left(\frac{1}{q(t)} \int_{0}^{t} f(s) h\left(u_{n}(s)\right) u_{n}^{\prime}(s) d s\right)^{1 / a}, \quad t \in(0, \infty), n \in \mathbb{N},
$$

we obtain

$$
k_{1}(\bar{u}(t)) \bar{u}^{\prime}(t)=\left(\frac{1}{q(t)} \int_{0}^{t} f(s) h(\bar{u}(s)) \bar{u}^{\prime}(s) d s\right)^{1 / a}, \quad t \in(0, \infty),
$$


and consequently, $\bar{u}$ is a solution of (1). Analogously we can prove that $\underline{u}$ is a solution of (1).

\section{Bounded and unbounded solutions}

TheOREm 4. Let assumptions $\left(\mathrm{H}_{1}\right)-\left(\mathrm{H}_{5}\right)$ be satisfied. Then

(i) some (and then any) solution of (1) is bounded if and only if

$$
\int_{0}^{\infty}\left(\frac{1}{q(t)}\right)^{1 / a} d t<\infty
$$

(ii) some (and then any) solution of (1) is unbounded if and only if

$$
\int_{0}^{\infty}\left(\frac{1}{q(t)}\right)^{1 / a} d t=\infty
$$

Pr o of. First note that either $\int_{0}^{\infty}(1 / q(t))^{1 / a} d t<\infty$ or $\int_{0}^{\infty}(1 / q(t))^{1 / a} d t$ $=\infty$. In the first case, by Lemma 3, any solution $u$ of (1) is bounded. Now assume $\int_{0}^{\infty}(1 / q(t))^{1 / a} d t=\infty$ and $u$ is a solution of (1). Then

$$
\begin{aligned}
\lim _{t \rightarrow \infty} \frac{\int_{0}^{t}\left(\frac{1}{q(s)} \int_{0}^{u(s)} f\left(u^{-1}(\tau)\right) h(\tau) d \tau\right)^{1 / a} d s}{\int_{0}^{t}\left(\frac{1}{q(s)}\right)^{1 / a} d s} \\
=\lim _{t \rightarrow \infty}\left(\int_{0}^{u(t)} f\left(u^{-1}(s)\right) h(s) d s\right)^{1 / a} \\
=\lim _{t \rightarrow \infty}\left(\int_{0}^{t} f(s) h(u(s)) u^{\prime}(s) d s\right)^{1 / a}>0
\end{aligned}
$$

and consequently,

$$
\lim _{t \rightarrow \infty} K_{1}(u(t))=\lim _{t \rightarrow \infty} \int_{0}^{t}\left(\frac{1}{q(s)} \int_{0}^{u(s)} f\left(u^{-1}(\tau)\right) h(\tau) d \tau\right)^{1 / a} d s=\infty .
$$

Hence $\lim _{t \rightarrow \infty} u(t)=\infty$ and $u$ is unbounded.

Let $u$ be a solution of (1). If $u$ is bounded, then $\int_{0}^{\infty}(1 / q(t))^{1 / a} d t<\infty$ since in the opposite case $u$ is unbounded by the first part of the proof. Analogously, $u$ unbounded implies $\int_{0}^{\infty}(1 / q(t))^{1 / a} d t=\infty$. 


\section{Uniqueness theorem}

ThEOREM 5. Let assumptions $\left(\mathrm{H}_{1}\right)-\left(\mathrm{H}_{5}\right)$ be satisfied. Moreover, assume that

$\left(\mathrm{H}_{6}\right) \quad$ There exist positive numbers $\varepsilon$ and $L$ such that

(i) $\left|f\left(t_{1}\right)-f\left(t_{2}\right)\right| \leq L\left|t_{1}-t_{2}\right|$ for all $t_{1}, t_{2} \in[0, \varepsilon]$,

(ii) the modulus of continuity $\gamma(t)\left(=\sup \left\{\left|\left(q\left(t_{1}\right)\right)^{1 / a}-\left(q\left(t_{2}\right)\right)^{1 / a}\right|\right.\right.$ : $\left.\left.t_{1}, t_{2} \in[0, \varepsilon],\left|t_{1}-t_{2}\right| \leq t\right\}\right)$ of $(q(t))^{1 / a}$ on $[0, \varepsilon]$ satisfies

$$
\limsup _{t \rightarrow 0_{+}} \frac{\gamma(t)}{t}<\infty
$$

Then (1) admits a unique solution.

Proof. By Theorem 1, there exists at least one solution of (1). Let $u_{1}$, $u_{2}$ be different solutions of $(1)$, say $u_{1}(t)<u_{2}(t)$ on $(0, \infty)$ (see Theorem 2$)$. According to the last part of the proof of Theorem 2 it is enough to show that $u_{1}(t)=u_{2}(t)$ on $\left[0, t_{0}\right]$ for a positive number $t_{0}$. Setting $A_{i}=\lim _{t \rightarrow \infty} u_{i}(t)$ and $w_{i}=u_{i}^{-1}(i=1,2)$, we see that $0<A_{1} \leq A_{2} \leq \infty, w_{i}:\left[0, A_{i}\right) \rightarrow \mathbb{R}_{+}$ are continuous strictly increasing functions and

$$
\begin{aligned}
& w_{i}(t)=\int_{0}^{t} k_{1}(s)\left(\frac{1}{q\left(w_{i}(s)\right)} \int_{0}^{s} f\left(w_{i}(\tau)\right) h(\tau) d \tau\right)^{-1 / a} d s, \\
& t \in\left[0, A_{i}\right), i=1,2 .
\end{aligned}
$$

Then (for $t \in\left[0, A_{1}\right)$ )

$$
\begin{aligned}
& (0 \leq) w_{1}(t)-w_{2}(t) \\
= & \int_{0}^{t} k_{1}(s)\left[\left(q\left(w_{1}(s)\right)\right)^{1 / a}-\left(q\left(w_{2}(s)\right)\right)^{1 / a}\right]\left(\int_{0}^{s} f\left(w_{2}(\tau)\right) h(\tau) d \tau\right)^{-1 / a} d s \\
& +\int_{0}^{t} \frac{k_{1}(s)\left(q\left(w_{1}(s)\right)\right)^{1 / a}}{\left(\int_{0}^{s} f\left(w_{1}(\tau)\right) h(\tau) d \tau \int_{0}^{s} f\left(w_{2}(\tau)\right) h(\tau) d \tau\right)^{1 / a}} \\
& \times\left[\left(\int_{0}^{s} f\left(w_{2}(\tau)\right) h(\tau) d \tau\right)^{1 / a}-\left(\int_{0}^{s} f\left(w_{1}(\tau)\right) h(\tau) d \tau\right)^{1 / a}\right] d s .
\end{aligned}
$$

Let $\varepsilon>0$ be as in assumption $\left(\mathrm{H}_{6}\right)$ and set $b=\min \left\{u_{1}(\varepsilon), \varepsilon\right\}, A=$ $\max \left\{(q(t))^{1 / a}: 0 \leq t \leq \varepsilon\right\}$ and $X(t)=\max \left\{w_{1}(s)-w_{2}(s): 0 \leq s \leq t\right\}$ for $t \in(0, b]$. Then $X$ is continuous nondecreasing, $X(0)=0, X(t)>0$ for $t \in(0, b]$ and $\left(\mathrm{cf} .\left(\mathrm{H}_{6}\right)\right)$

$$
\left|\left(q\left(w_{1}(t)\right)\right)^{1 / a}-\left(q\left(w_{2}(t)\right)\right)^{1 / a}\right| \leq \gamma(X(t)) \quad \text { for } t \in[0, b] .
$$


1. Let $a=1$. Then (cf. (14))

$$
\begin{aligned}
w_{1}(t)-w_{2}(t) \leq & \frac{1}{f(\varepsilon)} \int_{0}^{t} k(s) \gamma(X(s))(H(s))^{-1} d s \\
& +\frac{L}{(f(\varepsilon))^{2}} \int_{0}^{t} \frac{k(s) q\left(w_{1}(s)\right)}{(H(s))^{2}} \int_{0}^{s} h(\tau)\left(w_{1}(\tau)-w_{2}(\tau)\right) d \tau d s \\
\leq & \frac{1}{f(\varepsilon)} \gamma(X(t)) P(t)+\frac{L A}{(f(\varepsilon))^{2}} X(t) P(t), \quad t \in[0, b] .
\end{aligned}
$$

Hence

$$
X(t) \leq \frac{1}{f(\varepsilon)} \gamma(X(t)) P(t)+\frac{L A}{(f(\varepsilon))^{2}} X(t) P(t), \quad t \in[0, b]
$$

and

$$
1 \leq \frac{\gamma(X(t))}{f(\varepsilon) X(t)} P(t)+\frac{L A}{(f(\varepsilon))^{2}} P(t), \quad t \in(0, b] .
$$

Since

$$
\limsup _{t \rightarrow 0_{+}} \frac{\gamma(X(t))}{X(t)}=\limsup _{t \rightarrow 0_{+}} \frac{\gamma(t)}{t}<\infty \quad\left(\text { by }\left(\mathrm{H}_{6}\right)\right)
$$

and $\lim _{t \rightarrow 0_{+}} P(t)=0$, we get

$$
\lim _{t \rightarrow 0_{+}}\left[\frac{\gamma(X(t))}{f(\varepsilon) X(t)} P(t)+\frac{L A}{(f(\varepsilon))^{2}} P(t)\right]=0,
$$

which contradicts (15).

2. Let $a>1$. Then there is a positive integer $n$ such that $(n+1) / a>1$ and

$$
\begin{aligned}
& \left(\int_{0}^{t} f\left(w_{2}(s)\right) h(s) d s\right)^{(n+1) / a}-\left(\int_{0}^{t} f\left(w_{1}(s)\right) h(s) d s\right)^{(n+1) / a} \\
& =\left[\left(\int_{0}^{t} f\left(w_{2}(s)\right) h(s) d s\right)^{1 / a}-\left(\int_{0}^{t} f\left(w_{1}(s)\right) h(s) d s\right)^{1 / a}\right] \\
& \quad \times \sum_{k=0}^{n}\left(\int_{0}^{t} f\left(w_{2}(s)\right) h(s) d s\right)^{k / a}\left(\int_{0}^{t} f\left(w_{1}(s)\right) h(s) d s\right)^{(n-k) / a}
\end{aligned}
$$

By the Taylor formula,

$$
\begin{aligned}
\left(\int_{0}^{t} f\left(w_{2}(s)\right) h(s) d s\right)^{(n+1) / a}-\left(\int_{0}^{t} f\left(w_{1}(s)\right) h(s) d s\right)^{(n+1) / a} \\
=\frac{n+1}{a} \xi^{(n+1) / a-1} \int_{0}^{t}\left(f\left(w_{2}(s)\right)-f\left(w_{1}(s)\right)\right) h(s) d s
\end{aligned}
$$


where $\xi=\xi(t)$ lies in the interval with end points $\int_{0}^{t} f\left(w_{1}(s)\right) h(s) d s$, $\int_{0}^{t} f\left(w_{2}(s)\right) h(s) d s$, and thus (cf. (14) and (16))

$$
\begin{aligned}
w_{1}(t)- & w_{2}(t) \\
\leq & \int_{0}^{t} k_{1}(s) \gamma(X(s))(f(\varepsilon) H(s))^{-1 / a} d s \\
& +\int_{0}^{t} \frac{k_{1}(s)\left(q\left(w_{1}(s)\right)\right)^{1 / a}}{\left(\int_{0}^{s} f\left(w_{1}(\tau)\right) h(\tau) d \tau \int_{0}^{s} f\left(w_{2}(\tau)\right) h(\tau) d \tau\right)^{1 / a}} \\
& \times \frac{\left[\left(\int_{0}^{s} f\left(w_{2}(\tau)\right) h(\tau) d \tau\right)^{(n+1) / a}-\left(\int_{0}^{s} f\left(w_{1}(\tau)\right) h(\tau) d \tau\right)^{(n+1) / a}\right]}{\sum_{k=0}^{n}\left(\int_{0}^{s} f\left(w_{2}(\tau)\right) h(\tau) d \tau\right)^{k / a}\left(\int_{0}^{s} f\left(w_{1}(\tau)\right) h(\tau) d \tau\right)^{(n-k) / a}} d s \\
\leq & \gamma(X(t)) P(t)\left(\frac{1}{f(\varepsilon)}\right)^{1 / a}+\frac{n+1}{a} A\left(\frac{1}{f(\varepsilon)}\right)^{(n+2) / a} \\
& \times \int_{0}^{t} \frac{k_{1}(s) \xi^{(n+1) / a-1} \int_{0}^{s}\left(f\left(w_{2}(\tau)\right)-f\left(w_{1}(\tau)\right)\right) h(\tau) d \tau}{(n+1)(H(s))^{2 / a}(H(s))^{n / a}} d s \\
\leq & \gamma(X(t)) P(t)\left(\frac{1}{f(\varepsilon)}\right)^{1 / a}+\frac{A}{a}\left(\frac{1}{f(\varepsilon)}\right)^{(n+2) / a} L(f(0))^{(n+1) / a-1} \\
& \times \int_{0}^{t} \frac{k_{1}(s)(H(s))^{(n+1) / a} X(s)}{(H(s))^{(n+2) / a}} d s \leq \gamma(X(t)) P(t)\left(\frac{1}{f(\varepsilon)}\right)^{1 / a} \\
& +\frac{A}{a}\left(\frac{1}{f(\varepsilon)}\right)^{(n+2) / a}(f(0))^{(n+1) / a-1} L X(t) P(t)
\end{aligned}
$$

for $t \in[0, b]$ since $|\xi(t)| \leq f(0) H(t)$ on $[0, b]$. Then

$$
\begin{aligned}
X(t) \leq & \gamma(X(t)) P(t)\left(\frac{1}{f(\varepsilon)}\right)^{1 / a} \\
& +\frac{A}{a}\left(\frac{1}{f(\varepsilon)}\right)^{(n+2) / a}(f(0))^{(n+1) / a-1} L X(t) P(t),
\end{aligned}
$$

hence

$$
\begin{aligned}
1 \leq & \frac{\gamma(X(t))}{X(t)} P(t)\left(\frac{1}{f(\varepsilon)}\right)^{1 / a} \\
& +\frac{A}{a}\left(\frac{1}{f(\varepsilon)}\right)^{(n+2) / a}(f(0))^{(n+1) / a-1} L P(t)
\end{aligned}
$$

for $t \in(0, b]$, and since $\limsup \sup _{t \rightarrow 0_{+}} \gamma(X(t)) / X(t)<\infty$ and $\lim _{t \rightarrow 0_{+}} P(t)=0$, 
we get

$\lim _{t \rightarrow 0_{+}}\left[\frac{\gamma(X(t))}{X(t)} P(t)\left(\frac{1}{f(\varepsilon)}\right)^{1 / a}+\frac{A}{a}\left(\frac{1}{f(\varepsilon)}\right)^{(n+2) / a}(f(0))^{(n+1) / a-1} L P(t)\right]=0$,

which contradicts (17).

3. Let $a<1$. By the Taylor formula,

$$
\begin{aligned}
\left(\int_{0}^{t} f\left(w_{2}(s)\right) h(s) d s\right)^{1 / a}-\left(\int_{0}^{t} f\left(w_{1}(s)\right) h(s) d s\right)^{1 / a} \\
=\frac{\nu^{1 / a-1}}{a}\left(\int_{0}^{t} f\left(w_{2}(s)\right) h(s) d s-\int_{0}^{t} f\left(w_{1}(s)\right) h(s) d s\right)
\end{aligned}
$$

where $\nu=\nu(t)$ lies in the interval with end points $\int_{0}^{t} f\left(w_{2}(s)\right) h(s) d s$ and $\int_{0}^{t} f\left(w_{1}(s)\right) h(s) d s$, and using (14) we obtain

$w_{1}(t)-w_{2}(t)$

$$
\begin{aligned}
= & \gamma(X(t)) P(t)\left(\frac{1}{f(\varepsilon)}\right)^{1 / a}+\frac{A}{a}\left(\frac{1}{f(\varepsilon)}\right)^{2 / a}(f(0))^{1 / a-1} \\
& \times \int_{0}^{t} \frac{k_{1}(s)(H(s))^{1 / a-1}}{(H(s))^{2 / a}} \int_{0}^{s}\left(f\left(w_{2}(\tau)\right)-f\left(w_{1}(\tau)\right)\right) h(\tau) d \tau d s \\
\leq & \gamma(X(t)) P(t)\left(\frac{1}{f(\varepsilon)}\right)^{1 / a}+\frac{A}{a}\left(\frac{1}{f(\varepsilon)}\right)^{2 / a}(f(0))^{1 / a-1} L \int_{0}^{t} \frac{k_{1}(s) X(s)}{(H(s))^{1 / a}} d s \\
\leq & \gamma(X(t)) P(t)\left(\frac{1}{f(\varepsilon)}\right)^{1 / a}+\frac{A}{a}\left(\frac{1}{f(\varepsilon)}\right)^{2 / a}(f(0))^{1 / a-1} L X(t) P(t)
\end{aligned}
$$

for $t \in[0, b]$ since $|\nu(t)| \leq f(0) H(t)$ on $[0, b]$. Then

$$
\begin{array}{r}
X(t) \leq \gamma(X(t)) P(t)\left(\frac{1}{f(\varepsilon)}\right)^{1 / a}+\frac{A}{a}\left(\frac{1}{f(\varepsilon)}\right)^{2 / a}(f(0))^{1 / a-1} L X(t) P(t), \\
t \in[0, b],
\end{array}
$$

and hence

$$
1 \leq \frac{\gamma(X(t))}{X(t)} P(t)\left(\frac{1}{f(\varepsilon)}\right)^{1 / a}+\frac{A}{a}\left(\frac{1}{f(\varepsilon)}\right)^{2 / a}(f(0))^{1 / a-1} L P(t), \quad t \in(0, b],
$$

which contradicts

$$
\lim _{t \rightarrow 0_{+}}\left[\frac{\gamma(X(t))}{X(t)} P(t)\left(\frac{1}{f(\varepsilon)}\right)^{1 / a}+\frac{A}{a}\left(\frac{1}{f(\varepsilon)}\right)^{2 / a}(f(0))^{1 / a-1} L P(t)\right]=0 .
$$


6. Dependence of solutions on a parameter. Consider the differential equation

$$
\left(q(t) k(u)\left(u^{\prime}\right)^{a}\right)^{\prime}=\lambda f(t) h(u) u^{\prime}, \quad \lambda>0,
$$

depending on the positive parameter $\lambda$ with $q, k, f$ and $h$ satisfying assumptions $\left(\mathrm{H}_{1}\right)-\left(\mathrm{H}_{5}\right)$. Set

$$
\begin{aligned}
& \underline{\varphi}(t, \lambda)=P^{-1}\left(\int_{0}^{t}\left(\lambda \frac{f(s)}{q(s)}\right)^{1 / a} d s\right), \\
& \bar{\varphi}(t, \lambda)=P^{-1}\left(\int_{0}^{t}\left(\lambda \frac{f(0)}{q(s)}\right)^{1 / a} d s\right)
\end{aligned}
$$

for $(t, \lambda) \in \mathbb{R}_{+} \times(0, \infty)$. Denote by $u(t, \lambda)$ a solution of $\left(18_{\lambda}\right)$. By Theorem 3 (with $\lambda f$ instead of $f$ ), there exist solutions $\underline{u}(t, \lambda)$ and $\bar{u}(t, \lambda)$ of $\left(18_{\lambda}\right)$ such that

$$
\begin{aligned}
\underline{\varphi}(t, \lambda) \leq \underline{u}(t, \lambda) \leq u(t, \lambda) \leq \bar{u}(t, \lambda) \leq \bar{\varphi}(t, \lambda), & \\
& (t, \lambda) \in \mathbb{R}_{+} \times(0, \infty),
\end{aligned}
$$

for any solution $u(t, \lambda)$ of $\left(18_{\lambda}\right)$.

THEOREM 6. Let assumptions $\left(\mathrm{H}_{1}\right)-\left(\mathrm{H}_{5}\right)$ be satisfied. Then

$$
\bar{u}\left(t, \lambda_{1}\right)<\underline{u}\left(t, \lambda_{2}\right), \quad t \in(0, \infty),
$$

for any $0<\lambda_{1}<\lambda_{2}$.

Proof. Let $0<\lambda_{1}<\lambda_{2}$. Since

$$
\lim _{t \rightarrow 0_{+}} \frac{\int_{0}^{t}\left(\lambda_{2} \frac{f(s)}{q(s)}\right)^{1 / a} d s}{\int_{0}^{t}\left(\lambda_{1} \frac{f(0)}{q(s)}\right)^{1 / a} d s}=\lim _{t \rightarrow 0_{+}} \frac{\left(\lambda_{2} f(t)\right)^{1 / a}}{\left(\lambda_{1} f(0)\right)^{1 / a}}=\left(\lambda_{2} / \lambda_{1}\right)^{1 / a}>1
$$

there exists an $\varepsilon>0$ such that $\underline{\varphi}\left(t, \lambda_{2}\right)>\bar{\varphi}\left(t, \lambda_{1}\right)$ for $t \in(0, \varepsilon]$, and consequently,

$$
\bar{u}\left(t, \lambda_{1}\right)<\underline{u}\left(t, \lambda_{2}\right) \quad \text { for } t \in(0, \varepsilon]
$$

by (19). Assume $\bar{u}\left(t, \lambda_{1}\right)<\underline{u}\left(t, \lambda_{2}\right)$ on $\left(0, t_{0}\right)$ while $\bar{u}\left(t_{0}, \lambda_{1}\right)=\underline{u}\left(t_{0}, \lambda_{2}\right)$ for a $t_{0} \in(\varepsilon, \infty)$. Then 


$$
\begin{aligned}
0= & K_{1}\left(\underline{u}\left(t_{0}, \lambda_{2}\right)\right)-K_{1}\left(\bar{u}\left(t_{0}, \lambda_{1}\right)\right) \\
= & \int_{0}^{t_{0}}\left(\frac{\lambda_{2}}{q(t)} \int_{0}^{\left.\underline{u} t, \lambda_{2}\right)} f\left(\underline{u}^{-1}\left(s, \lambda_{2}\right)\right) h(s) d s\right)^{1 / a} d t \\
& -\int_{0}^{t_{0}}\left(\frac{\lambda_{1}}{q(t)} \int_{0}^{\bar{u}\left(t, \lambda_{1}\right)} f\left(\bar{u}^{-1}\left(s, \lambda_{1}\right)\right) h(s) d s\right)^{1 / a} d t,
\end{aligned}
$$

which contradicts

$$
\begin{aligned}
\left(\frac{\lambda_{2}}{q(t)} \int_{0}^{\underline{u}\left(t, \lambda_{2}\right)}\right. & \left.f\left(\underline{u}^{-1}\left(s, \lambda_{2}\right)\right) h(s) d s\right)^{1 / a}-\left(\frac{\lambda_{1}}{q(t)} \int_{0}^{\bar{u}\left(t, \lambda_{1}\right)} f\left(\bar{u}^{-1}\left(s, \lambda_{1}\right)\right) h(s) d s\right)^{1 / a} \\
> & \left(\frac{\lambda_{2}}{q(t)} \int_{0}^{\bar{u}\left(t, \lambda_{1}\right)} f\left(\bar{u}^{-1}\left(s, \lambda_{1}\right)\right) h(s) d s\right)^{1 / a} \\
& -\left(\frac{\lambda_{1}}{q(t)} \int_{0}^{\bar{u}\left(t, \lambda_{1}\right)} f\left(\bar{u}^{-1}\left(s, \lambda_{1}\right)\right) h(s) d s\right)^{1 / a}>0 \quad \text { for } 0<t \leq t_{0} .
\end{aligned}
$$

Corollary 2. Let assumptions $\left(\mathrm{H}_{1}\right)-\left(\mathrm{H}_{5}\right)$ be satisfied. Then there exists an at most countable set $\mathcal{R} \subset(0, \infty)$ such that equation $\left(18_{\lambda}\right)$ has a unique solution for every $\lambda \in(0, \infty)-\mathcal{R}$.

Proof. Let $t_{0} \in(0, \infty)$ and set $g(\lambda)=\underline{u}\left(t_{0}, \lambda\right)$ for $\lambda \in(0, \infty)$. Then $g$ is strictly increasing on $(0, \infty)$ by Theorem 6 , and

$$
\begin{aligned}
\lim _{\lambda \rightarrow \infty} g(\lambda) & =\lim _{\lambda \rightarrow \infty} \underline{u}\left(t_{0}, \lambda\right) \\
& \geq \lim _{\lambda \rightarrow \infty} \underline{\varphi}\left(t_{0}, \lambda\right)=\lim _{\lambda \rightarrow \infty} P^{-1}\left(\int_{0}^{t_{0}}\left(\lambda \frac{f(s)}{g(s)}\right)^{1 / a} d s\right)=\infty .
\end{aligned}
$$

Evidently, if $g$ is continuous at a point $\lambda=\lambda_{0}$ then $\left(18_{\lambda}\right)$ has a unique solution for $\lambda=\lambda_{0}$. For each $n \in \mathbb{N}$ denote by $\mathcal{R}_{n}$ the set of points of discontinuity of $g$ on the interval $[1 / n, n]$. By Theorem 1 of $[5$, p. 229], the set $\mathcal{R}_{n}$ is at most countable. Hence $\mathcal{R}=\bigcup_{n=1}^{\infty} \mathcal{R}_{n}$ is the set of points of discontinuity of $g$ and since $\mathcal{R}$ is at most countable, the proof of Corollary 2 is finished.

TheOREM 7. Let assumptions $\left(\mathrm{H}_{1}\right)-\left(\mathrm{H}_{6}\right)$ be satisfied and, moreover, $\int_{0}^{\infty}(1 / q(t))^{1 / a} d t<\infty$. Then for each $c \in(0, \infty)$ there exists a unique $\lambda_{c} \in(0, \infty)$ such that equation $\left(18_{\lambda}\right)$ for $\lambda=\lambda_{c}$ has a (necessarily unique) solution $u\left(t, \lambda_{c}\right)$ with

$$
\lim _{t \rightarrow \infty} u\left(t, \lambda_{c}\right)=c
$$


Proof. By Theorem 5, equation $\left(18_{\lambda}\right)$ has a unique solution $u(t, \lambda)$ for each $\lambda \in(0, \infty)$. This solution is strictly increasing (by Corollary 1 ) and bounded on $\mathbb{R}_{+}$(by Theorem 4). Define $g(\lambda)=\lim _{t \rightarrow \infty} u(t, \lambda)$ for all $\lambda>0$. The function $g:(0, \infty) \rightarrow(0, \infty)$ is increasing by Theorem 6 . To prove our theorem it is sufficient to show that $g$ is continuous, strictly increasing and maps $(0, \infty)$ onto itself. Assume $g\left(\lambda_{1}\right)=g\left(\lambda_{2}\right)$ for some $0<\lambda_{1}<\lambda_{2}$. Then $u\left(t, \lambda_{1}\right)<u\left(t, \lambda_{2}\right)$ on $(0, \infty)$ and thus

$$
\begin{aligned}
g\left(\lambda_{1}\right) & =\int_{0}^{\infty}\left(\frac{\lambda_{1}}{q(t)} \int_{0}^{u\left(t, \lambda_{1}\right)} f\left(u^{-1}\left(s, \lambda_{1}\right)\right) h(s) d s\right)^{1 / a} d t \\
& <\int_{0}^{\infty}\left(\frac{\lambda_{2}}{q(t)} \int_{0}^{u\left(t, \lambda_{2}\right)} f\left(u^{-1}\left(s, \lambda_{2}\right)\right) h(s) d s\right)^{1 / a} d t=g\left(\lambda_{2}\right),
\end{aligned}
$$

a contradiction. Assume

$$
\lim _{\lambda \rightarrow \lambda_{0+}} g(\lambda)-\lim _{\lambda \rightarrow \lambda_{0-}} g(\lambda)>0 \quad \text { for a } \lambda_{0} \in(0, \infty) .
$$

Set

$$
\alpha(t)=\lim _{\lambda \rightarrow \lambda_{0+}} u(t, \lambda), \quad \beta(t)=\lim _{\lambda \rightarrow \lambda_{0-}} u(t, \lambda) \quad \text { for } t \in \mathbb{R}_{+} .
$$

Then

$$
\liminf _{t \rightarrow \infty}(\alpha(t)-\beta(t))>0
$$

Using the Lebesgue dominated convergence theorem as $\lambda \rightarrow \lambda_{0+}$ and $\lambda \rightarrow$ $\lambda_{0-}$ in the equality

$$
\begin{aligned}
u(t, \lambda)=K_{1}^{-1}\left(\int_{0}^{t}\left(\frac{\lambda}{q(s)} \int_{0}^{u(s, \lambda)} f\left(u^{-1}(\tau, \lambda)\right) h(\tau) d \tau\right)^{1 / a} d s\right) & \\
& (t, \lambda) \in \mathbb{R}_{+} \times(0, \infty),
\end{aligned}
$$

we see (cf. Lemma 2) that $\alpha$ and $\beta$ are solutions of $\left(18_{\lambda_{0}}\right)$. Consequently, $\alpha(t)=\beta(t)=u\left(t, \lambda_{0}\right)$ for $t \in \mathbb{R}_{+}$, which contradicts (21). Finally,

$$
\begin{aligned}
& \lim _{\lambda \rightarrow \infty} \lim _{t \rightarrow \infty} \underline{\varphi}(t, \lambda)=\lim _{\lambda \rightarrow \infty} P^{-1}\left(\int_{0}^{\infty}\left(\frac{\lambda f(s)}{q(s)}\right)^{1 / a} d s\right)=\infty, \\
& \lim _{\lambda \rightarrow 0_{+}} \lim _{t \rightarrow \infty} \bar{\varphi}(t, \lambda)=\lim _{\lambda \rightarrow 0_{+}} P^{-1}\left(\int_{0}^{\infty}\left(\frac{\lambda f(0)}{q(s)}\right)^{1 / a} d s\right)=0,
\end{aligned}
$$

since $\lim _{t \rightarrow \infty} P^{-1}(t)=\infty, \lim _{t \rightarrow 0_{+}} P^{-1}(t)=0$,

$$
0<\int_{0}^{\infty}\left(\frac{f(s)}{q(s)}\right)^{1 / a} d s<\int_{0}^{\infty}\left(\frac{f(0)}{q(s)}\right)^{1 / a} d s<\infty
$$

and therefore (cf. (19)) $\lim _{\lambda \rightarrow \infty} g(\lambda)=\infty$ and $\lim _{\lambda \rightarrow 0_{+}} g(\lambda)=0$. 


\section{References}

[1] F. A. Atkinson and L. A. Peletier, Similarity profiles of flows through porous media, Arch. Rational Mech. Anal. 42 (1971), 369-379.

[2] —, - Similarity solutions of the nonlinear diffusion equation, ibid. 54 (1974), 373392 .

[3] J. Bear, D. Zaslavsky and S. Irmay, Physical Principles of Water Percolation and Seepage, UNESCO, 1968.

[4] J. Goncerzewicz, H. Marcinkowska, W. Okrasiński and K. Tabisz, On the percolation of water from a cylindrical reservoir into the surrounding soil, Zastos. Mat. 16 (1978), 249-261.

[5] P. Natanson, Theorie der Funktionen einer reellen Veränderlichen, AkademieVerlag, Berlin, 1969.

[6] W. Okrasiński, Integral equations methods in the theory of the water percolation, in: Mathematical Methods in Fluid Mechanics, Proc. Conf. Oberwolfach, 1981, Band 24, P. Lang, Frankfurt/M, 1982, 167-176.

[7] -, On a nonlinear ordinary differential equation, Ann. Polon. Math. 49 (1989), 237245 .

[8] S. Staněk, Nonnegative solutions of a class of second order nonlinear differential equations, ibid. 57 (1992), 71-82.

[9] -, Qualitative behavior of a class of second order nonlinear differential equations on the halfline, ibid. 58 (1993), 65-83.

DEPARTMENT OF MATHEMATICAL ANALYSIS

FACULTY OF SCIENCE, PALACKÝ UNIVERSITY

TOMKOVA 40

77906 OLOMOUC, CZECH REPUBLIC 